\title{
Propriedades físico-químicas e tecnológicas da farinha do resíduo de açaí e sua utilização
}

\author{
Physico-chemical and technological properties of acai residue flour and its use \\ Propiedades físico-químicas y tecnológicas de la harina de resíduos de açaí y su uso
}

Recebido: 31/03/2021 | Revisado: 10/04/2021 | Aceito: 19/04/2021 | Publicado: 03/05/2021

\author{
Mikaelly Veiga Borges \\ ORCID: https://orcid.org/0000-0001-5632-4387 \\ Universidade Federal de Goiás, Brasil \\ E-mail: mikaelly.vb@gmail.com \\ Eduardo Brauno de Sousa \\ ORCID: https://orcid.org/0000-0002-2856-9528 \\ Universidade Federal de Goiás, Brasil \\ E-mail: edubrauno@gmail.com \\ Miriam Fontes Araújo Silveira \\ ORCID: https://orcid.org/0000-0002-0191-8628 \\ Universidade Federal de Goiás, Brasil \\ E-mail: miriamfas.ufg@gmail.com \\ Adriana Régia Marques de Souza \\ ORCID: https://orcid.org/0000-0002-0608-9988 \\ Universidade Federal de Goiás, Brasil \\ E-mail: adriana_regia_souza@ufg.br \\ Vania Maria Alves \\ ORCID: https://orcid.org/0000-0003-3949-6691 \\ Universidade Federal do Tocantins, Brasil \\ E-mail:vanialvees@gmail.com \\ Luana Beatriz Moreira Nunes \\ ORCID: https://orcid.org/ 0000-0002-1380-7028 \\ Universidade Federal de Goiás, Brasil \\ E-mail: nunesluana633@gmail.com \\ Samara Kelly Amaral Barros \\ ORCID: https://orcid.org/0000-0002-7090-8650 \\ Universidade Federal do Tocantins, Brasil \\ E-mail: aramasly@gmail.com
}

\section{Resumo}

São inúmeros os resíduos gerados pelas indústrias de alimentos, o que leva a busca por alternativas de aproveitamento e desenvolvimento de subprodutos. Os resíduos de açaí, por sua vez, conhecidos popularmente como 'borra', são obtidos em grande quantidade, tendo em vista, o montante processado nas agroindústrias. O presente trabalho teve como objetivo estudar as propriedades físico-químicas e tecnológicas da farinha do resíduo de açaí (FRA), e avaliar sua utilização, como ingrediente, no processamento de quibe. Foram realizadas as seguintes análises físico-químicas: umidade, proteínas, lipídios, cinzas, carboidratos, valor energético, fibras, $\mathrm{pH}$ e acidez titulável. As propriedades tecnológicas estudadas foram: solubilidade em água, índice de absorção em água e índice de absorção em óleo. Para avaliação sensorial, três formulações de quibe foram desenvolvidas, com diferentes concentrações da FRA (05, 10 e 15\%). A FRA apresentou altos teores de fibras e elevado valor energético, bem como propriedades tecnológicas satisfatórias para utilizá-la, como ingrediente, em formulações de produtos cárneos. As formulações de quibe com 05 e 10\% de FRA foram as preferidas pelos consumidores, tornando seu acréscimo uma alternativa viável para as indústrias de alimentos

Palavras-chave: Subproduto; Aproveitamento; Quibe.

\begin{abstract}
There are countless residues generated by the food industries, which leads to the search for alternatives for the use and development of by-products. Açaí residues, in turn, popularly known as 'sludge', are obtained in large quantities, in view of the amount processed in agro-industries. The present work aimed to study the physico-chemical and technological properties of açaí residue flour (FRA), and to evaluate its use, as an ingredient, in the processing of kibbeh. The following physical-chemical analyzes were carried out: moisture, proteins, lipids, ash, carbohydrates, energy value, fibers, $\mathrm{pH}$ and acidity. The technological properties studied were: water solubility, water absorption index and oil absorption index. For sensory evaluation, three kibbeh formulations were developed, with different concentrations of FRA $(05,10$ and 15\%). The FRA presented high levels of fibers and high caloric value, as well as
\end{abstract}


satisfactory technological properties to use it, as an ingredient, in formulations of meat products. Kibbeh formulations with 05 and 10\%FRA were preferred by consumers, making their addition a viable alternative for the food industries.

Keywords: By-product; Use; Fried meat balls.

\section{Resumen}

Son innumerables los residuos generados por las industrias alimentarias, lo que lleva a la búsqueda de alternativas para el uso y desarrollo de subproductos. Los residuos de açaí, a su vez, conocidos popularmente como 'lodos', se obtienen en grandes cantidades, dada la cantidad procesada en las agroindustrias. Este trabajo tuvo como objetivo estudiar las propiedades físico-químicas y tecnológicas de la harina de residuo de açaí (FRA) y evaluar su uso como ingrediente en el procesamiento de kibbeh. Se realizaron los siguientes análisis físico-químicos: humedad, proteínas, lípidos, cenizas, carbohidratos, valor energético, fibras, $\mathrm{pH}$ y acidez. Las propiedades tecnológicas estudiadas fueron: solubilidad en agua, índice de absorción de agua e índice de absorción de aceite. Para la evaluación sensorial se desarrollaron tres formulaciones de kibbeh, con diferentes concentraciones de FRA (05, 10 y 15\%). FRA presentó altos niveles de fibras y alto valor calórico, así como propiedades tecnológicas satisfactorias para utilizarlo, como ingrediente, en formulaciones de productos cárnicos. Los consumidores prefirieron las formulaciones de Kibbeh con 05 y 10\% de FRA, lo que hace que su adición sea una alternativa viable para las Industrias alimentarias.

Palabras clave: Subproducto; Utilización; Kibbeh.

\section{Introdução}

Segundo a Organização das Nações Unidas (ONU), cerca de 931 milhões de toneladas de alimentos foram desperdiçados no ano de 2019, o que equivale a 17\% do total de alimentos disponíveis aos consumidores (ONU, 2021).

Os elevados índices de perdas e desperdícios, gerados pelas indústrias de alimentos, levam à busca por alternativas de aproveitamento e desenvolvimento de novos produtos (DNP), que possam ser acrescidos de nutrientes e apelos funcionais (Pereira, et al., 2005). Dentro do DNP, a farinha de subprodutos tem sido bastante utilizada para fortificar produtos de panificação e contribuir para a diminuição e aproveitamento de resíduos agroindustriais (Larrosa \& Otero, 2021).

O aproveitamento desses subprodutos, além dos fatores sustentáveis, ambientais e econômicos, está associado aos altos teores de fibras encontrados em sua composição. Estudos relacionam a ingestão alimentar de fibras com a manutenção da saúde e prevenção de doenças (Monteiro, et al., 2020).

As indústrias de alimentos, aproveitando a oportunidade, colocaram no mercado vários produtos enriquecidos em fibra, visando atender à demanda crescente de indivíduos interessados em hábitos saudáveis (Stella, 2007). Entre os produtos em ascensão, devido a uma alta procura comercial, tem-se o açaí, uma das frutas que se destaca no Brasil, com grande crescimento em sua produção e com geração de resíduos cada vez maior (Buratto; Cocero, \& Martin, 2021).

O açaí é o fruto do açaizeiro (Euterpe oleracea Mart.), contém elevado teor de antocianinas, responsáveis por suas características funcionais, além de apresentar um alto valor energético. É rico em fibras, vitamina E, proteínas, minerais e ácidos graxos essenciais, como Ômega-6 e Ômega-9 (Maia; Souza \& Lima, 2007). Os resíduos de açaí, conhecidos popularmente como 'borra', são obtidos em grande quantidade, tendo em vista o montante processado nas agroindústrias, que são, muitas vezes, considerados lixo orgânico, porém apresentam elevados teores de nutrientes, podendo ser incorporados na elaboração de outros produtos. Seria oportuno efetuar estudos sobre a borra do açaí, para criar possibilidades de valorização desse subproduto (Lins, 2008).

Nesse contexto, tem-se a possibilidade de incorporar o resíduo de açaí na fabricação de quibe, substituindo parcialmente o trigo. O quibe é um prato originalmente do oriente médio e, segundo a Instrução Normativa $n^{\circ} 20$, de 31 de julho de 2000 (Brasil, 2000), entende-se por quibe (kibe) o produto cárneo industrializado, obtido de carne bovina ou ovina, moída, adicionado com trigo integral, acrescido de ingredientes. Dentre os componentes obrigatórios tem-se a carne, o trigo integral e a água, já os opcionais são: sal, gordura vegetal ou animal, proteína de origem animal, recheios, condimentos, aromas e especiarias (Vitorassi, 2012). O trigo integral é um alimento rico em proteína, gordura, vitamina E e do complexo B, bem como minerais, em particular, cálcio, fósforo, potássio, magnésio, enxofre e ferro. 
O quibe é um salgado popular, facilmente encontrado em quiosques e comércios, comercializado cru, cozido, assado ou frito. É muito consumido devido ao seu alto teor energético, entretanto não apresenta fibras em sua constituição (Barbosa; Sales \& Dias, 2011).

Diante do exposto, o objetivo deste trabalho foi estudar as propriedades físico-químicas e tecnológicas da farinha do resíduo de açaí (FRA), bem como avaliar sua utilização, como ingrediente, no processamento de quibe.

\section{Metodologia}

O experimento foi conduzido nos Laboratórios de Análise de Alimentos, do Setor de Engenharia de Alimentos, da Escola de Agronomia (EA), e no Laboratório de Química e Bioquímica de Alimentos, da Faculdade de Farmácia (FF), da Universidade Federal de Goiás (UFG).

\subsection{Obtenção da Farinha do Resíduo de Açaí (FRA)}

Os resíduos de açaí (Euterpe oleracea Mart), obtidos após a despolpa do fruto, realizada na Universidade Federal do Tocantins (UFT), foram transportados em caixas isotérmicas em ônibus comercial. A matéria-prima chegou congelada no Setor de Engenharia de Alimentos/EA/UFG, iniciando-se o processo de secagem em estufa de circulação forçada de ar a $60^{\circ} \mathrm{C}$, até a obtenção de um produto com umidade máxima final de 15\%. Após secos, os resíduos de açaí foram triturados em moinho de facas (willye START FT 50), para obtenção da farinha com granulometria fina $(25,40 \mathrm{~mm})$. A farinha foi acondicionada em sacos de polietileno de baixa densidade, devidamente etiquetada e armazenada à temperatura ambiente, para posterior realização das análises.

\subsection{Análises Físico-Químicas da FRA}

\subsubsection{Composição proximal e valor energético}

Os teores de umidade, cinzas e proteínas foram determinados de acordo com AOAC (2012), os lipídios totais pelo método Bligh \& Dyer (1959) e os carboidratos estimados por diferença, subtraindo-se de 100\% a soma dos valores obtidos para as determinações anteriores.

O valor energético foi calculado utilizando-se os fatores de conversão de Atwater (carboidrato, 4,0 Kcal/g; lipídeos, 9,0 Kcal/g; proteínas, 4,0 Kcal/g), expresso em Kcal (Mendez, et al., 1995).

\subsubsection{Fibras}

As fibras foram determinadas pelo método gravimétrico - enzimático, com utilização de enzimas ( $\alpha$-amilase, protease e amiloglicosidase), com resultados expressos em g/100 g (AOAC, 2012).

\subsubsection{Acidez Total Titulável (ATT)}

A acidez total titulável foi realizada por meio de titulação, com solução de hidróxido de sódio $(\mathrm{NaOH}) 0,1 \mathrm{M}$, expresso em ácido cítrico em g/100g (AOAC, 2012).

\subsection{4 $\mathrm{pH}$}

O potencial hidrogeniônico $(\mathrm{pH})$ foi medido em potenciômetro de bancada, marca HANNA ${ }^{\circledR}$ Instruments, modelo HI 9224, calibrado com soluções de pH 7,0 e 4,0, segundo a AOAC (2012). 


\subsection{Propriedades tecnológicas da FRA}

\subsection{1 Índice de solubilidade em água (ISA)}

Para determinação do ISA foi utilizada a metodologia descrita por Santana (2005). O cálculo foi feito pela Equação 1.

$$
\operatorname{ISA}(\%)=[\mathrm{A} / \mathrm{B}] \mathrm{x} 100 \quad \text { (Equação 1) }
$$

$\mathrm{A}=$ massa da amostra seca;

$\mathrm{B}=$ massa da amostra

\subsection{2 Índice de absorção em água (IAA)}

Para determinação do IAA foi utilizada metodologia descrita por Santana (2005), com modificações. Amostras de dois gramas de farinha foram suspensas em $25 \mathrm{~mL}$ de água destilada a $50{ }^{\circ} \mathrm{C}$, colocadas em tubos de centrífuga de $100 \mathrm{~mL}$, previamente pesados, e submetidos à agitação por 30 minutos. Após, foram centrifugados a $2500 \mathrm{rpm}$ por 10 minutos a $10^{\circ} \mathrm{C}$. O sobrenadante foi transferido para placa de petri, de peso conhecido, e o tubo, contendo amostra final com água, foi pesado. O IAA, em gramas de amostra hidratada por gramas de amostra seca, foi obtido pela Equação 2.

$$
\operatorname{IAA}(\%)=[\mathrm{A} / \mathrm{B}] \times 10 \quad \text { (Equação 2) }
$$

$\mathrm{A}=$ massa da amostra hidratada;

$\mathrm{B}=$ massa da amostra desidratada.

\subsection{3 Índice de absorção em óleo (IAO)}

Para determinação do IAO foi empregada metodologia descrita por Santana (2005), com modificações. Amostras contendo um grama de farinha foram suspensas em $10 \mathrm{~mL}$ de óleo de canola a $25{ }^{\circ} \mathrm{C}$ e colocadas em tubos de centrífugas de 50 $\mathrm{mL}$, previamente pesados. Em seguida, foram agitados por 30 minutos e centrifugados a $2500 \mathrm{rpm}$ por 10 minutos a $25^{\circ} \mathrm{C}$. $\mathrm{O}$ líquido sobrenadante foi descartado e o IAO, em gramas de amostra com óleo por grama de amostra seca, foi obtido pela Equação 3.

$$
\operatorname{IAO}(\%)=[\mathrm{A} / \mathrm{B}] \mathrm{x} 100 \quad \text { (Equação 3) }
$$

$\mathrm{A}=$ massa da amostra insolúvel;

$\mathrm{B}=$ massa da amostra desidratada.

\subsection{Desenvolvimento de quibe com FRA}

Foram desenvolvidas 3 (três) formulações de quibe, com 05, 10 e 15\% de farinha de resíduo de açaí (FRA), em substituição ao trigo para quibe (Tabela 1). As concentrações dos demais ingredientes não foram alteradas nas formulações.

Foi considerada como $100 \%$ da formulação a soma das porcentagens das matérias-primas (carne moída, trigo para quibe e FRA). A porcentagem dos demais ingredientes (água, cebola, alho, pimenta, manteiga e sal) foi calculada em relação à soma das matérias-primas. 
Tabela 1. Formulações de quibe com farinha de resíduo de açaí (FRA).

\begin{tabular}{cccc}
\hline $\begin{array}{c}\text { Ingredientes } \\
(\%)\end{array}$ & $\begin{array}{c}\text { Formulação 1 } \\
(\%)\end{array}$ & $\begin{array}{c}\text { Formulação 2 } \\
(\%)\end{array}$ & $\begin{array}{c}\text { Formulação 3 } \\
(\%)\end{array}$ \\
\hline Carne moída & 50 & 50 & 50 \\
Trigo para quibe & 45 & 40 & 35 \\
FRA & 05 & 10 & 15 \\
Água & 37,50 & 37,50 & 37,50 \\
Cebola & 5 & 5 & 5 \\
Alho & 0,75 & 0,75 & 0,75 \\
Pimenta do reino & 0,50 & 0,50 & 0,50 \\
Manteiga & 4 & 4 & 4 \\
Sal & 0,30 & 0,30 & 0,30 \\
\hline
\end{tabular}

Fonte: Própria (2021).

Em balança semianalítica (0,01g), marca Even ${ }^{\circledR}$, modelo BL-3200AS-BI, a FRA e o trigo para quibe foram pesados, conforme cada formulação, e homogeneizados. Em seguida, foram hidratados com água fervente, durante 30 minutos. Posteriormente, a carne moída (acém) e os demais ingredientes foram pesados, homogeneizados e divididos em três porções iguais, para elaboração das formulações. As porções foram misturadas às farinhas hidratadas e colocadas em fôrmas. Após, foram levadas ao forno a $200^{\circ} \mathrm{C}$ por 15 minutos, cobertas com papel alumínio, e por 25 minutos sem papel alumínio.

\subsection{Análise sensorial}

Foi realizado um teste de ordenação de preferência, com 70 provadores não treinados, com idade entre 13 e 65 anos. Os provadores receberam três amostras codificadas com números aleatórios de três dígitos para avaliá-las e ordená-las em ordem crescente de preferência, onde a nota 1 indicava a amostra mais preferida e a nota 3 , a menos preferida. O projeto foi aprovado no Comitê de Ética e Pesquisa da Universidade Federal do Tocantins com o número 20699519.3.0000.5519.

\subsection{Análise estatística}

Os resultados foram expressos pela média e pelo desvio padrão de quinze medidas de uma amostra de farinha e analisados por estatística descritiva. $\mathrm{O}$ experimento foi realizado com cinco repetições, em triplicata.

$\mathrm{Na}$ análise sensorial, as ordenações de preferência, atribuídas por cada provador, foram somadas e analisadas de acordo com o Teste de Friedman (5\% de significância), na tabela de Newell e MacFarlane, segundo Ferreira, et al. (2000).

\section{Resultados e Discussão}

\subsection{Análises físico-químicas da FRA}

Os resultados das análises físico-químicas da FRA estão apresentados na Tabela 2. 
Tabela 2. Caracterização físico-química da farinha do resíduo de açaí (FRA).

\begin{tabular}{cc}
\hline Parâmetros & FRA \\
\hline Umidade (\%) & $9,06 \pm 0,79$ \\
Cinzas (\%) & $1,31 \pm 0,13$ \\
Proteínas (\%) & $6,65 \pm 0,01$ \\
Lipídios (\%) & $1,57 \pm 0,10$ \\
Carboidratos (\%) & $81,41 \pm 0,47$ \\
VE (Kcal) & $366,37 \pm 0,56$ \\
Fibras Totais (\%) & $47,09 \pm 0,28$ \\
pH & $4,30 \pm 0,79$ \\
ATT (g de ácido cítrico/100g) & $1,22 \pm 0,01$ \\
\hline
\end{tabular}

Fonte: Própria (2021).

O resultado do teor de umidade $(9,06 \%)$ mostra que a FRA apresenta características favoráveis ao armazenamento, garantindo segurança e estabilidade ao produto (Ladeira \& Pena, 2011). O valor encontrado está dentro do máximo permitido para farinha (15\%), segundo legislação brasileira em vigor (Brasil, 2005), indicando baixa disponibilidade de água para a ocorrência de reações químicas e crescimento microbiano (Fernandes, et al., 2008). Martins, et al. (2020) encontraram valores próximos para umidade em farinha de caroço de açaí (11,70\%). Valores superiores ao de $14 \%$ em farinhas tendem a formar grumos com outros ingredientes, prejudicando a homogeneização (Fernandes, et al. 2008).

O teor de cinzas da FRA foi de 1,31\%, próximo ao encontrado por Arruda, et al. (2018) em farinha de resíduo de semente de açaí (1,22\%). Segundo Oliveira, Pinto \& Rezende (2017), em termos nutricionais, maiores teores de cinzas significam elevados teores de minerais, mas para estudos de produção de energia, o baixo teor de cinza é importante para um bom rendimento energético (Sato, et al., 2019).

O teor de proteínas na FRA (6,65\%) foi semelhante ao encontrado por Silva, et al. (2018) em farinha de semente de açaí $(6,70 \%)$, apresentando um produto pouco proteico, o que pode ser explicado pelo fato da farinha ser um produto mais energético e não proteico (Santos, 2011).

A análise de lipídeos totais indicou um valor de 1,57\% na FRA, abaixo do encontrado por Martins, et al. (2020), de 3,76\%, em farinha do resíduo do caroço de açaí. Fasolin, Almeida \& Netto-Oliveira (2007) encontraram teor de lipídios de $1,89 \%$ em farinha de banana verde, próximos ao deste trabalho.

O conteúdo de cinzas, proteínas e lipídios influenciam nas propriedades tecnológicas da farinha, sendo que menores concentrações destes componentes indicam um maior grau de pureza dos carboidratos. Além disso, os lipídios contribuem para o baixo inchamento dos grânulos de amido (Zhang, et al., 2017).

A FRA apresentou alto teor de carboidratos em sua composição $(81,41 \%)$, podendo ser utilizada como alimento de elevado valor energético, seja pelo consumo in natura ou incorporada como ingrediente em novos produtos (Abud \& Narain, 2009). De acordo com Lima (2009), valores elevados de carboidratos, provavelmente, se devem às macromoléculas, como a celulose e o amido presentes na borra residual de açaí.

Destaca-se que dentre os constituintes do carboidrato tem-se amido, celulose, pectina e outros biopolímeros presentes em cascas de vegetais. Assim, os teores de fibras da FRA estão incluídos nos valores encontrados para carboidratos. César 
(2007) encontrou 25,22\% de fibras em polpa de açaí, valor menor ao obtido na FRA (47,09\%). Este resultado indica que para o enriquecimento nutricional a partir de fibras, é mais vantajoso o uso da FRA.

Tendo a FRA um alto teor de fibras, aponta-se um potencial para utilização como ingrediente em formulações de diversos produtos com o objetivo de promover benefícios à saúde (Souza, et al., 2018). Larrosa \& Otero (2021) evidenciam que farinhas elaboradas a partir de resíduos são boas fontes de fibra, na qual apresentam propriedades funcionais.

O valor energético encontrado na FRA (366,37 Kcal) apresentou-se superior ao de polpas de açaí de onze pontos comerciais (113,49 Kcal), analisadas por Aquino, et al. (2019). Assim, sugere-se a utilização da FRA como ingrediente em formulações para o enriquecimento energético de produtos processados.

O pH encontrado na FRA foi de 4,30, levemente ácida. Santos, et al. (2008) encontraram valores de pH em polpas de açaí variando de 3,55 a 4,89. O açaí é uma fruta pouco ácida e sua acidez não varia com o tempo. Variações de pH, segundo Oliveira; Farias \& Pena (2007), são devidas aos ácidos orgânicos presentes no fruto. Baixos valores de pH dificultam o crescimento de microrganismos e favorecem a vida útil do produto (Castro, et al., 2017).

A ATT da FRA foi de 1,22 g de ácido cítrico/100g, abaixo do máximo estabelecido pelo MAPA, que é de 3,2 g/100 g (Brasil, 2000). Os ácidos orgânicos influenciam nas características sensoriais e estabilidade do alimento. Uma das principais funções do ácido cítrico é a sua ação conservante (Gonçalves, et al., 2018).

\subsection{Propriedades tecnológicas da FRA}

Na Tabela 3 estão apresentados os resultados obtidos das propriedades tecnológicas da FRA. Estas propriedades influenciam a aparência física e o comportamento das biomoléculas, relacionando seu desempenho em produtos destinados à alimentação.

Tabela 3. Propriedades tecnológicas da farinha do resíduo de açaí (FRA).

\begin{tabular}{cc}
\hline Propriedades & Valores \\
\hline Solubilidade em água (\%) & $3,94 \pm 1,54$ \\
Índice de absorção em água $\left(\mathrm{g} \cdot \mathrm{g}^{-1}\right)$ & $3,12 \pm 0,25$ \\
Índice de absorção em óleo $\left(\mathrm{g} \cdot \mathrm{g}^{-1}\right)$ & $2,47 \pm 0,15$ \\
\hline
\end{tabular}

Fonte: Própria (2021).

O resultado encontrado para solubilidade em água (SA) foi de 3,94\%, considerado baixo. Segundo Tavares, et al. (2012), a SA está relacionada com o teor de umidade da matéria-prima, sendo que altos valores são encontrados em alimentos com umidade superior a $22 \mathrm{~g} / 100 \mathrm{~g}$.

Bastos (2012), ao estudar as aplicações de resíduos, cita que valores elevados de solubilidade são importantes para misturas dextrinizadas, como molhos, sopas, entre outros. Porém, para produtos cárneos não é necessária alta SA, pois, nestes, procura-se uma textura mais firme e que não se dissolva em altas temperaturas.

O índice de absorção em água (IAA) da FRA foi de 3,12 g. $\mathrm{g}^{-1}$, superior ao encontrado por Martins (2019) em farinha de araçá, de 1,45 g.g ${ }^{-1}$. O IAA é uma propriedade importante no estudo da utilização da FRA como ingrediente em formulações de produtos cárneos, pois permite a adição de água a fim de facilitar o manuseio da massa e evitar seu ressecamento. No caso do processamento do quibe, essa característica é muito importante na fase de hidratação, pois a absorção de água permite inchaço e expansão de partículas (Santana; Filho \& Egea, 2017). 
A FRA apresentou índice de absorção em óleo (IAO) de 2,47 g.g $\mathrm{g}^{-1}$. Khattab \& Arntfield (2009), trabalhando com produtos à base de milho e canola, encontraram valores de 2,01 g.g-1 e 2,09 g.g ${ }^{-1}$, respectivamente. Castilho, et al. (2010) também encontraram valores menores no IAO em farinha de tremoço doce $\left(1,2-1,3 \mathrm{~g} \cdot \mathrm{g}^{-1}\right)$ e farinha de feijão guandu (2,0-2,1 g. $\mathrm{g}^{-1}$ ), considerado valores satisfatórios para desempenho da funcionalidade em alimentos industrializados. A absorção em óleo favorece a retenção de sabor e torna agradável ao paladar; contribuindo, assim, com a aceitação sensorial do produto (Seena \& Sridhar, 2005).

Baú, et al. (2012) observaram que, além das propriedades de hidratação, as fibras possuem a capacidade de reter o óleo, podendo utilizá-las em alimentos ricos em gordura e emulsões, pois facilitam a estabilização (Grigelmo; Carreiras \& Martín, 1999). A absorção de óleo é uma propriedade de grande importância para farinhas, pois está relacionada ao aumento do paladar e à retenção de sabor (Kinsella, 1976).

\subsection{Análise Sensorial}

No teste de ordenação, a soma das posições indica que os menores valores são as amostras que apareceram mais vezes em primeiro lugar no teste de ordenação, ou seja, a mais preferida (Tabela 4). Os resultados do teste de ordenação de preferência mostraram que os produtos com menores teores de FRA (5\% e 10\%) foram preferidos pelos provadores. O atributo "textura" foi o mais mencionado como razão pela preferência.

Tabela 4. Análise sensorial de preferência de quibes com diferentes teores de farinha do resíduo de açaí (FRA).

\begin{tabular}{cc}
\hline FRA $(\%)$ & Soma das posições ordenadas \\
\hline 05 & $120^{\mathrm{A}}$ \\
10 & $139^{\mathrm{AB}}$ \\
15 & $161^{\mathrm{B}}$ \\
\hline
\end{tabular}

Valores seguidos das mesmas letras não diferem estatisticamente entre si, pelo teste de Friedman $(p>0,05)$. Fonte: Própria (2021).

Os produtos com adição de 05 e $10 \%$ de FRA foram os preferidos pelos provadores, não houve diferença significativa entre essas amostras ( $>>0,05)$. As menores concentrações de FRA estão associadas a maiores índices de aceitabilidade, como descrito em outros trabalhos com incorporação de resíduos (Lima, et al., 2019, Silva, et al., 2018), possivelmente por se assemelhar ao produto tradicional.

As amostras com 05 e $15 \%$ de FRA diferiram estatisticamente, sendo que o produto com maior teor foi o que apresentou menor preferência. Os provadores atribuíram características indesejáveis ao produto, como textura arenosa, granulosa e de maior mastigabilidade, que pode ser explicado pelo alto conteúdo de fibras. Zambelli (2014) ressalta que ao incorporar mais ingredientes funcionais, observa-se um aumento de mastigabilidade, exigindo maior energia para mastigar o produto.

Em contraste, os provadores que preferiram o quibe com acréscimo de 15\% de FRA, justificaram a escolha devido ao "melhor sabor" e "produto mais temperado". Esses atributos podem estar associados ao desempenho das fibras que exercem capacidade de retenção de matérias graxas e de água, justificado pelo índice satisfatório de absorção em óleo (IAO), responsável pela intensificação do sabor no produto (FIB, 2014).

Percebe-se que o intervalo de preferência do consumidor se concentrou nas amostras com teores de FRA menores ou iguais a $10 \%$. 


\section{Conclusão}

A farinha do resíduo de açaí (FRA) apresentou alto conteúdo de fibra alimentar, podendo ser empregada em substituição parcial ao trigo para quibe, proporcionando melhorias nutricionais. Sensorialmente, a FRA proporcionou mais sabor ao produto incorporado, ao mesmo tempo deixando-o mais granuloso. Sendo assim, a FRA se apresenta como uma fonte valiosa de fibras alimentares, a ser acrescentada em outras farinhas, pois seus componentes auxiliam nas características finais do produto alimentício ao qual foi incorporado.

\section{Referências}

Abud, A. K. S. \& Narain, N. (2009). Incorporação da farinha de resíduo do processamento de polpa de fruta em biscoitos: uma alternativa de combate ao desperdício. Brazilian Journal of Food Technology, 12(4), 257-265.

Álvares, V. S. (2013). Caracterização físico-química de farinhas de mandioca (Manihot Esculenta Crantz) dos povos indígenas Kaxinawa. Anais do Congresso Brasileiro de Mandioca, Salvador, BA, Brasil, 791.

AOAC. (2012). Association of Official Analytical Chemists. Official methods of analysis. 19a ed. Washington: Editorial Board. 3000p.

Aquino C. M., Moreira, L. F., de Lima Mendes, A. H., dos Santos, S. M. L., \& de Sousa Monte, A. L. (2019). (2019). Avaliação físico-química e microbiológica de açaí (Euterpe oleracea) congelado pronto para o consumo comercializado em Limoeiro do Norte-Ceará. Biota Amazônia, 9(3), 35-40.

Arruda J. C. B., Fonseca, L. A. B. D., Pinto, L. C. P., Pinheiro, H. C. D. O., Monteiro, B. T. O., Manno, M. C., Lima, K. R. S., \& Lima, A. R. D. (2018). Açaí seed bran in the feed of slow-growth broilers. Acta Amazonica, 48(4), 298-303.

Barbosa, E. S.; Sales, S. S. \& Dias, D. S. (2011). Frequência do consumo de alimentos de origem animal de alunos com idade escolar na cidade de Montes Claros. Anais do Fórum de Pesquisa, Ensino, Extensão e Gestão da Unimontes, Montes Claros, MG, Brasil, 16.

Bastos, G. M. (2012). Resíduos da industrialização de batata: aplicação na produção de farinhas, snacks, farinhas pré-gelatinizadas e massa alimentícia fresca sem glúten, Dissertação de Mestrado, Universidade Federal de Goiás, Goiânia, GO, Brasil.

Baú T. R., da Silva, L. C., Garcia, S., \& Ida, E. I. (2012). Propriedades funcionais tecnológicas das fibras de soja, aveia e trigo e produtos de soja com adição de fibras e fermentados com cultura de kefir. Semina: Ciências Agrárias, 33(2), 3093-3102.

Bligh, E. G. \& Dyer, W. J. (1959). A rapid method of total lipid extraction and purification. Canadian Journal of Biochemistry and Physiology, 37(8), 911917.

Buratto, R. T., Cocero, M. J. e Martin, A. (2021). Characterization of industrial acai pulp residues and valorization by microwave-assisted extraction. Chemical Engineering and Processing - Process Intensification. Elsevier B.V., 160(108269). doi: 10.1016/j.cep.2020.108265.

Brasil (2005). Agência Nacional de Vigilância Sanitária. Resolução da Diretoria Colegiada - RDC No 263 , de 23 de setembro de 2005 . Regulamento Técnico para produtos de cereais, amidos, farinhas e farelos. Diário Oficial da União. Brasília-DF. 2005.

Brasil (2000). Instrução Normativa ${ }^{\circ}$ 01, de 07 de janeiro de 2000. Regulamento Técnico Geral para fixação dos padrões de identidade e qualidade para polpa de fruta. Diário Oficial da União. Brasília- DF, 2000.

Castro, D. S. D., Oliveira, T. K. B. D., Lemos, D. M., Rocha, A. P. T., \& Almeida, R. D. (2017). Efeito da temperatura sobre a composição físico-química e compostos bioativos de farinha de taro obtida em leito de jorro. Brazilian Journal of Food Technology, 20 , e2016060.

Castilho, F; Fontanari, G. G. \& Batistuti, J. P. (2010). Avaliação de algumas propriedades funcionais das farinhas de tremoço doce (Lupinus albus) e feijão guandu (Cajanus cajan (L.) Millsp) e sua utilização na produção de fiambre. Ciência e Tecnologia de Alimentos, 30(1), 68-75.

César, L. T. (2007). Obtenção de suco clarificado de Açaí (Euterpe oleracea Mart.) com utilização de pectinase e quitosana. Dissertação de Mestrado, Universidade Federal do Ceará, Fortaleza, CE, Brasil.

Fasolin, L. H.; Almeida, G. C. \& Netto-Oliveira, E. R. (2007). Biscoitos produzidos com farinha de banana: avaliações química, física e sensorial. Ciência e Tecnologia de Alimentos, 27(3), 524-529.

Fellows, P. J. (2006). Tecnologia do processamento de alimentos: princípios e prática. Porto Alegre: Artmed.

Fernandes, A. F., Pereira, J., Germani, R., \& Oiano-Neto, J. (2008). Efeito da substituição parcial da farinha de trigo por farinha de casca de batata (Solanum Tuberosum Lineu). Ciência e Tecnologia de Alimentos, 28(suplemento), 56-65.

Ferreira, V., Almeida, T., Pettinelli, M., Silva, M., Chaves, J., \& Barbosa, E. (2000). Análise sensorial: testes discriminativos e afetivos. Campinas, SP: Sociedade Brasileira de Ciência e Tecnologia de Alimentos. 127 p. (Manual: Série Qualidade).

FIB - Food Ingredients Brasil. (2014). Fibras. Recuperado em 20 de junho, 2020, de https://revista-fi.com.br/.

Garcia, M. F. (2019). Brasil desperdiça 26,3 milhões de toneladas de alimentos por ano. Observatório do Terceiro Setor. Recuperado em 23 de março, 2020, de https://observatorio3setor.org.br/noticias/brasil-desperdica-26-milhoes-de-toneladas-de-alimentos-por-ano/attachment/desperdicio-de-alimentos-brasil-1/. 
Gonçalves, A. D. A., Arruda, M. R. A. D., Melo, F. L. D., \& Padilha, F. M. Q. D. H. (2018). Processamento e caracterização físico-química da farinha de berinjela (Solanum melongena L.): farinha de berinjela: produção e caracterização físico-química. Tese de Doutorado, Faculdade Pernambucana de Saúde, Recife, PE, Brasil.

Grigelmo, M. N.; Carreras, B. E. \& Martín B. O. (1999). Development Of high-fruit-dietary-fibre muffins. European Food Research and Technology, 210(2), $123-128$

Kinsella, J. E. (1976). Functional properties of proteins in foods: A survey. Critical Reviews in Food Science and Nutrition, 7(3), 219-280.

Khattab, R. Y. \& Arntfield, S. D. (2009). Functional properties of raw and processed canola meal. Food Science and Technology, 42, 1119-1124.

Ladeira, T. M. S. \& Pena, R. S. (2011). Propriedades físico-químicas e tecnológicas dos polvilhos azedos de três cultivares de mandioca. Alimentos e Nutrição. $22(4), 631-640$

Larrosa, A. P. Q. e Otero, D. M. (2021). Flour made from fruit by-products: Characteristics, processing conditions, and applications. Journal of Food Processing and Preservation. Blackwell Publishing Ltd, p. e15398. doi: 10.1111/jfpp.15398.

Lima, H. T. (2009). Aproveitamento de resíduos agroindustriais (borra de açaí e glicerol) na elaboração de biscoito. Dissertação de Mestrado. Universidade Federal do Pará, Belém. PA, Brasil.

Lins, K. J. C. (2008). Avaliação das características físico-químicas da borra resultante do processamento industrial de açaí (Euterpe Oleracea Mart.) e de seu uso para fins alimentícios. Trabalho de conclusão de curso (TCC) em Engenharia de Alimentos. Universidade Federal do Pará, Belém. PA, Brasil.

Maia, G. A.; Sousa, P. H. M. \& Lima, A. S. (2007). Principais frutas tropicais para processamento de polpas, sucos e néctares. Fortaleza: Ed. UFC.

Martins, L. H. S., Konagano, E. M. H., da Silva Souza, R. L. L., \& Lopes, A. S. (2020). Análise físico-química de diferentes resíduos agroindustriais para possível utilização na indústria. Brazilian Journal of Development, 6(2), 6936-6948.

Martins, T. S. (2019). Produção de coproduto de araçá (Psidium cattleianum): Características da farinha e sua aplicação como novo ingrediente na indústria de panificação. Trabalho de conclusão de curso (TCC) em Tecnologia de Alimentos. Universidade Federal da Paraíba, João Pessoa, PB, Brasil.

Mendez, M. H., Derivi, S. C. N., Rodrigues, M. C. R., \& Fernandes, M. L. (1995). Tabela de composição de alimentos. Niterói: EDUFF (Editora da Universidade Federal Fluminense).

Monteiro, S. A., Barbosa, M. M., da Silva, F. F. M., Bezerra, R. F., \& da Silva Maia, K. (2020). Preparation, phytochemical and bromatological evaluation of flour obtained from the acerola (Malpighia punicifolia) agroindustrial residue with potential use as fiber source, LWT. Academic Press, 134, p. 110142. doi: 10.1016/j.lwt.2020.110142.

Oliveira, C. F. D.; Pinto, E. G. \& Rezende, P. L. R. (2017). Compostos Bioativos de extratos de pequi de diferentes regiões do cerrado. Enciclopédia Biosfera, 14(25), 1799-1813.

Oliveira, M. S. P; Farias J. T. \& Pena R. S. (2007). Açaí: técnicas de cultivo e processamento. Fortaleza: Instituto Frutal.

ONU (2021). Organização das Nações Unidas. Relatório Índice de Desperdício de Alimentos 2021.

Pereira, C. A., CARLI, L. D., Beux, S., Santos, M. S., Busato, S. B., Kobelnik, M., \& Barana, A. C. (2005). Utilização de farinha obtida a partir de rejeito de batata na elaboração de biscoitos. Ciência Agronômica de Engenharia, 11(1), 19-26.

Santana, G. S.; Filho, J. G. de O. \& Egea, M. B. Características tecnológicas de farinhas vegetais comerciais. Revista de Agricultura Neotropical, $4(2), 2017$.

Santana, M. F. S. (2005). Caracterização físico-química de fibra alimentar de laranja e maracujá, Tese de Doutorado, Universidade Estadual de Campinas, Campinas, SP, Brasil.

Santos, C. A., Ribeiro, R. C., SILVA, E. D., Silva, N., SILVA, B. D., SILVA, G. D., \& Barros, B. C. V. (2011). Elaboração de biscoito de farinha de Buriti (Mauritia flexuosa L.f) com e sem adição de aveia (Avena sativa L.) Revista Brasileira de Tecnologia Agroindustrial, 5(1), $262-273$.

Santos G. M., Maia, G. A., Sousa, P. H. M., Costa, J. M. C., Figueiredo, R. W., \& Prado, G. M. (2008). Correlação entre atividade antioxidante e compostos bioativos de polpas comerciais de açaí (Euterpe oleracea Mart). Archivos Latinoamericanos de Nutrición, 58(2), 187-192,

Sarantópoulos, C. I. G. L. \& Oliveira, E. C. (2001). Requisitos de conservação de alimentos em embalagens flexíveis. Campinas: CETEA/ITAL.

Sato, M. K., Lima, H. V., Costa, A. N., Rodrigues, S., Pedroso, A. J. S., \& Maia, C. M. B. F. (2019). Biochar from Acai agroindustry waste: Study of pyrolysis conditions. Waste Management, 96, 158-167.

Seena, S. \& Sridhar, K. R. (2005). Physicochemical functional and cooking properties of under explored legumes, Canavalia of the southwest coast of Índia. Food Research International, 38(7), 803-814.

Silva, J. P., Oliveira, E. R. N., Pereira, S. C. M., \& Monteiro, A. R. G. (2018). Avaliação Físico-Química e Sensorial de Pães Produzidos com Substituição Parcial de Farinha de Trigo por Farinha de Banana Verde. Revista Brasileira de Pesquisa em Alimentos. 5 (3), 1 - 7.

Silva, R. C. Batista, A., da Costa, D. C. F., Moura-Nunes, N., Koury, J. C., Costa, C. A., Resende, A. C., \& Daleprane, J. B. (2018). Açai (Euterpe oleracea Mart.) seed flour prevents obesity-induced hepatic steatosis regulating lipid metabolism by increasing cholesterol excretion in high-fat diet-fed mice. Food Research International. Elsevier Ltd, 111, p. 408-415. doi: 10.1016/j.foodres.2018.05.043.

Souza, J. O., Camilloto, G. P., \& Cruz, R. S. (2018). Biscoitos tipo amanteigado incorporado com farinha de caroço de Açaí. Anais do Seminário de Iniciação Científica, Feira de Santana, BA, Brasil. 
Research, Society and Development, v. 10, n. 5, e17810514517, 2021

(CC BY 4.0) | ISSN 2525-3409 | DOI: http://dx.doi.org/10.33448/rsd-v10i5.14517

Stella, R. (2007) Fibras para seu intestino. Recuperado em 27 fevereiro, 2020, de http:www.uol.com.br/cyberdiet/colunas/010921_nut_fibra_intestino.htm.

Tavares, J. S., Soares Júnior, M. S., Becker, F. S., \& Costa, E. E. D. (2012). Mudanças funcionais de farinha de arroz torrada com micro-ondas em função do teor de umidade e do tempo de processamento. Ciência Rural, 42(6). 1102-1109.

Vitorassi, D. C. (2012.). Desenvolvimento de quibe de carne mecanicamente separada de tilápia com adição de linhaça (Linum usitatissimum L.) para inserção na merenda escolar. Trabalho de conclusão de curso em Tecnólogo em Alimentos. Universidade Tecnológica Federal do Paraná, Medianeira, PR, Brasil.

Zambelli, R. A. (2014). Desenvolvimento de massas congeladas de pães tipo forma contendo ingredientes funcionais. Dissertação de Mestrado. Universidade Federal do Ceará, Fortaleza, CE, Brasil.

Zhang, L., Li, G., Wang, S., Yao, W., \& Zhu, F. (2017). Physicochemical properties of maca starch. Food Chemistry, 218 (Supplement C), 56-63. 